\title{
Production of (anti-)(hyper-)nuclei at LHC energies with ALICE
}

\author{
Maximiliano Puccio ${ }^{1,2, \star}$ for the ALICE collaboration \\ ${ }^{1}$ Dipartimento di Fisica, Università degli Studi di Torino \\ ${ }^{2}$ Istituto Nazionale di Fisica Nucleare, Sezione di Torino
}

\begin{abstract}
The ALICE experiment at the LHC has measured a variety of (anti-)(hyper-)nuclei produced in $\mathrm{Pb}-\mathrm{Pb}$ collisions at $\sqrt{s_{\mathrm{NN}}}=5.02 \mathrm{TeV}$ and at $2.76 \mathrm{TeV}$. In addition, a large sample of high quality data was collected in pp collisions at $\sqrt{s}=7$ $\mathrm{TeV}$ and $13 \mathrm{TeV}$ and in $\mathrm{p}-\mathrm{Pb}$ collisions at $\sqrt{s_{\mathrm{NN}}}=5.02 \mathrm{TeV}$. These data are used to study the production of different (anti-)(hyper-)nuclei in the collisions, namely (anti-)deuteron, $(\text { anti-) })^{3} \mathrm{He}$, (anti-)alpha and (anti-) ${ }_{\Lambda}^{3} \mathrm{H}$. The identification of these (anti-)(hyper-)nuclei is based on the energy loss measurement in the Time Projection Chamber and the velocity measurement in the Time-Of-Flight detector. In addition, the Inner Tracking System is used to distinguish secondary vertices originating from weak decays from the primary vertex. New results on deuteron production as a function of multiplicity in $\mathrm{pp}, \mathrm{p}-\mathrm{Pb}$ and $\mathrm{Pb}-\mathrm{Pb}$ collisions will be presented, as well as the measurement of ${ }^{3} \mathrm{He}$ in $\mathrm{p}-\mathrm{Pb}$ and $\mathrm{Pb}-$ $\mathrm{Pb}$ collisions. Special emphasis will be given to the new results of the $(\text { anti- })_{\Lambda}^{3} \mathrm{H}$ in its charged-two-body decay mode. The large variety of measurements at different energies and system sizes constrains the production models of light flavour baryon clusters, in particular those based on coalescence and the statistical hadronisation approaches.
\end{abstract}

\section{Introduction}

The nuclei, anti-nuclei and hyper-nuclei formation mechanism in high energy collisions is still unknown. Thanks to the LHC which provides $\mathrm{pp}, \mathrm{p}-\mathrm{Pb}$ and $\mathrm{Pb}-\mathrm{Pb}$ collisions at the highest energy ever reached in the laboratory, the ALICE experiment is able to shed light on the phenomenology of (anti-)(hyper-)nuclei production. The latest results on the production spectra of (anti-)deuteron and (anti-) $)^{3} \mathrm{He}$ in pp collisions at $\sqrt{s}=13 \mathrm{TeV}$ and $\mathrm{Pb}-\mathrm{Pb}$ collisions at $\sqrt{s_{\mathrm{NN}}}=5.02 \mathrm{TeV}$ will be discussed here. The results are compared with the expectation from the statistical hadronisation and the hadron coalescence models. Finally the new measurement of the (anti-) ${ }_{\Lambda}^{3} \mathrm{H}$ lifetime by the ALICE experiment, which is the most precise ever performed, will be discussed.

\section{Analyses details}

The key features that allow ALICE to measure (anti-)(hyper-)nuclei are the precise vertexing, tracking capabilities and the redundancy of particle identification detectors.

Tracking and vertexing are performed using the Inner Tracking Systems (ITS), a silicon tracker featuring 6 cylindrical layers, and the Time Projection Chamber (TPC) [1]. Thanks to the extended

\footnotetext{
^e-mail: maximiliano.puccio@cern.ch
} 

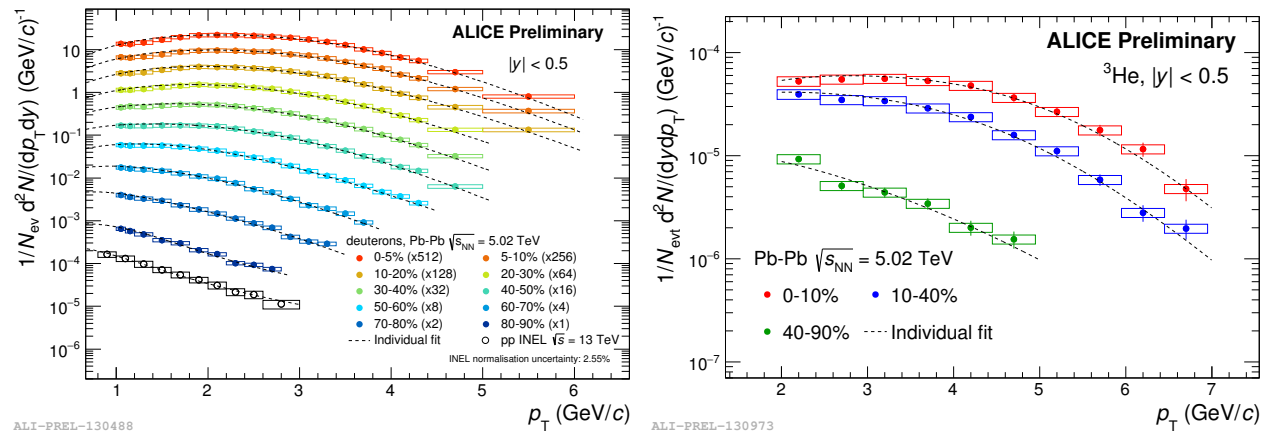

Figure 1. Transverse momentum spectra of deuterons (on the left) and ${ }^{3} \mathrm{He}$ (on the right) in $\mathrm{Pb}-\mathrm{Pb}$ collisions at $\sqrt{s_{\mathrm{NN}}}=5.02 \mathrm{TeV}$. The $p_{\mathrm{T}}$ production spectrum of deuterons in $\mathrm{pp}$ at $\sqrt{s}=13 \mathrm{TeV}$ is also reported in the left panel. Statistical uncertainties are represented as vertical bars whereas boxes represent the systematic ones. The dashed lines represent the fit to the individual spectra (Blast Wave function in $\mathrm{Pb}-\mathrm{Pb}$ [8] and the Lévy-Tsallis in $\mathrm{pp}[9])$ to extrapolate the production yield in the unmeasured $p_{\mathrm{T}}$ regions.

lever arm and a maximum solenoidal magnetic field of $0.5 \mathrm{~T}$, the momentum resolution is better than $1 \%$ for particles with $p \leq 100 \mathrm{GeV} / c$ [2]. Tracks reconstructed with points in the innermost ITS layer have a pointing resolution better than $300 \mu \mathrm{m}$ [2]. For (anti-)(hyper-)nuclei identification the TPC specific energy loss signal $(\mathrm{d} E / \mathrm{d} x)$ is used to clearly separate nuclei with $\mathrm{Z}=2$ over the full measured momentum range from the bulk of the produced charged particles. Lighter nuclei with $\mathrm{Z}=1$ can be identified clearly by means of the TPC $\mathrm{d} E / \mathrm{d} x$ only at low momenta (e.g. $p \leq 1.4 \mathrm{GeV} / c$ for (anti-)deuterons), but thanks to the Time Of Flight detector (TOF) it is possible to identify, for instance, (anti-)deuterons up to $p_{\mathrm{T}}=6 \mathrm{GeV} / c$ using a statistical unfolding technique [3]. In order to identify (anti-) ${ }_{\Lambda}^{3} \mathrm{H}$ decaying in (anti- $)^{3} \mathrm{He}$ and $\pi^{-(+)}$it is necessary to reconstruct its decay vertex. For each possible pair of (anti- $)^{3} \mathrm{He}$ and $\pi^{-(+)}$the point of closest approach between the two tracks is computed and a set of topological selection is applied. A pair of (anti- $)^{3} \mathrm{He}$ and $\pi^{-(+)}$that passes all these selections is considered to be a (anti- ${ }_{\Lambda}^{3} \mathrm{H}$ candidate. Finally a two components fit is performed to extract the yield of (anti-) ${ }_{\Lambda}^{3} \mathrm{H}$, similarly to what is illustrated in [4].

\section{Results}

The production spectra of deuterons in $\mathrm{Pb}-\mathrm{Pb}$ collisions at $\sqrt{s_{\mathrm{NN}}}=5.02 \mathrm{TeV}$ and in pp collisions at $\sqrt{s}=13 \mathrm{TeV}$ are shown in Figure 1 (left). The typical hardening of the spectra with increasing centrality, already observed for lighter particles $[5,6]$ and for deuterons $[3,7]$ at lower energies, is visible here and in the ${ }^{3} \mathrm{He}$ spectra (right panel of Figure 1): this pattern suggests an increasing radial flow with increasing centrality. Moreover, the ratio between the production spectra of nuclei and their anti-matter partners is compatible with unity [10], like for the other baryon over anti-baryon ratios at the LHC energies. This observation is compatible with expectations from the statistical hadronisation [11] and the hadron coalescence models [12]. In both approaches the abundances of a (anti-)nuclide $X$ with mass number $A$ are coupled to those of (anti-)protons according to the following relation: $\bar{X} / X \approx(\bar{p} / p)^{A}$. This expectation for the coalescence model holds only if the spectra of (anti-)neutrons are compatible with those of (anti-)protons. Under this assumption it is also possible to compute the coalescence parameter $B_{A}$ which is defined for a nuclide with mass number $A$ as the 

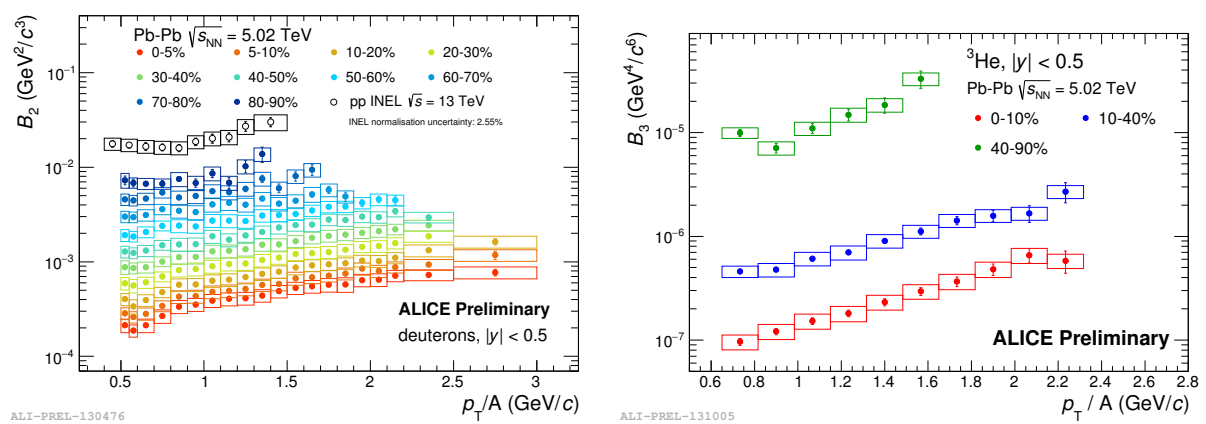

Figure 2. Coalescence parameters of deuterons $\left(B_{2}\right.$, left panel) and ${ }^{3} \mathrm{He}\left(B_{3}\right.$, right panel) measured in $\mathrm{Pb}-\mathrm{Pb}$ collisions at $\sqrt{s_{\mathrm{NN}}}=5.02 \mathrm{TeV}$. The $B_{2}$ measured in pp collisions at $\sqrt{s}=13 \mathrm{TeV}$ is also shown in the left panel. Statistical uncertainties are represented as vertical bars whereas boxes represent the systematic ones. The ALICE preliminary proton $p_{\mathrm{T}}$-production spectra [6] are used to compute the coalescence parameters.

ratio between the nuclide invariant production spectrum and the proton spectrum to the power of $A$. Figure 2 shows the coalescence parameters for deuterons and ${ }^{3} \mathrm{He}$. In the simple hadron coalescence approach the coalescence parameter is assumed to be momentum independent. However, the presence of correlations in the proton and neutron initial emission, such as jets or common emission radius from the thermal source [13], can introduce a momentum dependence similar to the measured $B_{A}$.

Another observable qualitatively described by the simple hadron coalescence is the deuteron over proton ratio as a function of multiplicity in small systems, see Figure 3. As more protons and neutrons are created in events with increasing multiplicities, the probability of finding two nucleons close in momentum space and consequently the probability of forming a deuteron increase in small systems. However, this scaling breaks in semi-central $\mathrm{Pb}-\mathrm{Pb}$ collisions where the deuteron over proton ratio as a function of multiplicity becomes flatter. A flat nucleus over proton ratio as a function of multiplicity, observed also in the case of ${ }^{3} \mathrm{He}$ shown in Figure 3, is expected if the statistical hadronisation is the underlying production mechanism in nucleus nucleus collisions. At very high multiplicity the measurements of the deuteron over proton ratios in $\mathrm{Pb}-\mathrm{Pb}$ collisions at $\sqrt{s_{\mathrm{NN}}}=2.76 \mathrm{TeV}$ and $\sqrt{s_{\mathrm{NN}}}=$ $5.02 \mathrm{TeV}$ show a hint of suppression. This kind of behaviour might point to the presence of a hadronic rescattering phase that might break nuclei, similar to what is proposed in [14].

Finally, the ALICE collaboration has been able to perform one of the most precise measurements of the ${ }_{\Lambda}^{3} \mathrm{H}$ lifetime using the $\mathrm{Pb}-\mathrm{Pb}$ data sample at $\sqrt{s_{\mathrm{NN}}}=5.02 \mathrm{TeV}$ collected in 2015. The lifetime was measured analysing the ${ }_{\Lambda}^{3} \mathrm{H} \rightarrow{ }^{3} \mathrm{He}+\pi^{-}$and the ${ }_{\bar{\Lambda}}^{3} \overline{\mathrm{H}} \rightarrow{ }^{3} \overline{\mathrm{He}}+\pi^{+}$decays in different $\mathrm{c} t$ intervals and performing an exponential fit to the obtained $\mathrm{d} N / \mathrm{d}(\mathrm{c} t)$ distribution, similarly to what has been done in [4]. The preliminary measured value $\tau=237_{-36}^{+33}$ (stat.) \pm 17 (syst.) ps is compatible with both the previously computed world average $\left(\tau=\left(216_{-16}^{+18}\right) \mathrm{ps}\right.$, [4]) and with the free $\Lambda$ lifetime.

\section{Conclusions}

The harvest of results from the LHC Run 2 data in the (anti-)nuclei sector just started. The new results at the current LHC top energies confirm the picture depicted by the Run 1 results [3, 7]: at the LHC energies the nuclei production mechanisms in small systems and in $\mathrm{Pb}-\mathrm{Pb}$ seem to be different. $\mathrm{On}$ one hand the simple hadron coalescence describes qualitatively the measurements of deuteron over proton ratio and $B_{2}$ up to peripheral $\mathrm{Pb}-\mathrm{Pb}$ collisions. On the other hand the steady rise of the $B_{2}$ with 

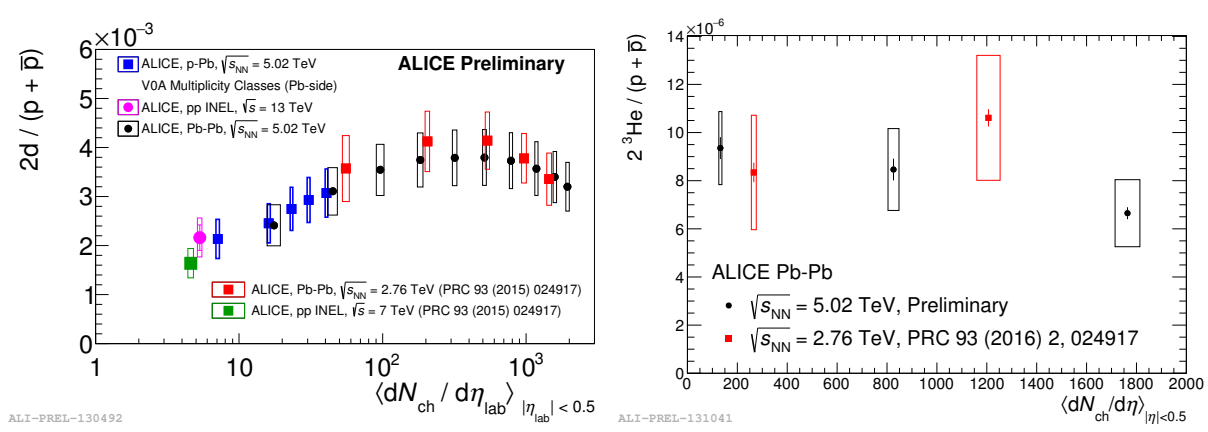

Figure 3. Deuteron (left panel) and ${ }^{3} \mathrm{He}$ (right panel) over proton ratios as a function of the charged particle multiplicity in the laboratory in different collision systems. Statistical uncertainties are represented as vertical lines whereas boxes represent the systematic ones.

momentum and the flattening of the deuteron over proton ratio in mid-central and central $\mathrm{Pb}-\mathrm{Pb}$ collisions require additional assumptions to reconcile the coalescence expectations with ALICE results. The larger data sample expected in the upcoming $2018 \mathrm{~Pb}-\mathrm{Pb}$ run will help to refine the results for ${ }^{3} \mathrm{He}$ and possibly extend the measurements to heavier nuclei to test the model predictions with increased precision. However, hadron coalescence might not be the production mechanism of (anti-)nuclei in $\mathrm{Pb}-\mathrm{Pb}$ collisions: the flat $\mathrm{d} / \mathrm{p}$ ratio starting from mid-central collisions is in agreement with the expectations of the statistical hadronisation model. Further studies on the systematic uncertainties will help to establish whether the decrease of the $\mathrm{d} / \mathrm{p}$ ratio in very central $\mathrm{Pb}-\mathrm{Pb}$ collision is significant.

In the strange nuclear sector, the new ALICE measurement of the ${ }_{\Lambda}^{3} \mathrm{H}$ lifetime is compatible with both the current world average and with the theoretical expectation (i.e. the free $\Lambda$ lifetime [15]).

\section{References}

[1] K. Aamodt et al. (ALICE), JINST 3, S08002 (2008)

[2] B.B. Abelev et al. (ALICE), Int. J. Mod. Phys. A29, 1430044 (2014), 1402.4476

[3] S. Acharya et al. (ALICE) (2017), 1707.07304

[4] J. Adam et al. (ALICE), Phys. Lett. B754, 360 (2016), 1506.08453

[5] J. Adam et al. (ALICE), Phys. Rev. C93, 034913 (2016), 1506.07287

[6] N. Jacazio (ALICE) (2017), 1704.06030

[7] J. Adam et al. (ALICE), Phys. Rev. C93, 024917 (2016), 1506.08951

[8] E. Schnedermann, J. Sollfrank, U.W. Heinz, Phys. Rev. C48, 2462 (1993), nucl-th/9307020

[9] C. Tsallis, J. Statist. Phys. 52, 479 (1988)

[10] ALICE (2017), ALICE-PUBLIC-2017-006, http://cds. cern.ch/record/2272148

[11] A. Andronic, P. Braun-Munzinger, J. Stachel, H. Stöcker, Phys. Lett. B 697, 203 (2011), 1010.2995

[12] J.I. Kapusta, Phys. Rev. C21, 1301 (1980)

[13] R. Scheibl, U.W. Heinz, Phys. Rev. C59, 1585 (1999), nucl-th/9809092

[14] F. Becattini, E. Grossi, M. Bleicher, J. Steinheimer, R. Stock, Phys. Rev. C90, 054907 (2014), 1405.0710

[15] R.J. Prem, P.H. Steinberg, Phys. Rev. 136, B1803 (1964) 